\title{
In Silico Analysis of Bacteriocins from Lactic Acid Bacteria Against SARS-CoV-2
}

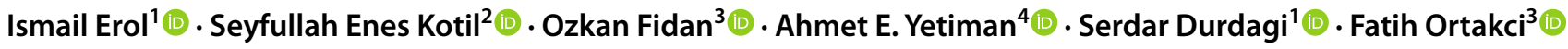

Accepted: 11 November 2021 / Published online: 27 November 2021

(c) The Author(s), under exclusive licence to Springer Science+Business Media, LLC, part of Springer Nature 2021

\begin{abstract}
The COVID-19 pandemic caused by a novel coronavirus (SARS-CoV-2) is a serious health concern in the twenty-first century for scientists, health workers, and all humans. The absence of specific biotherapeutics requires new strategies to prevent the spread and prophylaxis of the novel virus and its variants. The SARS-CoV-2 virus shows pathogenesis by entering the host cells via spike protein and Angiotensin-Converting Enzyme 2 receptor protein. Thus, the present study aims to compute the binding energies between a wide range of bacteriocins with receptor-binding domain (RBD) on spike proteins of wild type (WT) and beta variant (lineage B.1.351). Molecular docking analyses were performed to evaluate binding energies. Upon achieving the best bio-peptides with the highest docking scores, further molecular dynamics (MD) simulations were performed to validate the structure and interaction stability. Protein-protein docking of the chosen 22 biopeptides with WT-RBD showed docking scores lower than $-7.9 \mathrm{kcal} / \mathrm{mol}$. Pediocin PA-1 and salivaricin P showed the lowest (best) docking scores of $-12 \mathrm{kcal} / \mathrm{mol}$. Pediocin PA-1, salivaricin B, and salivaricin P showed a remarkable increase in the double mutant's predicted binding affinity with $-13.8 \mathrm{kcal} / \mathrm{mol},-13.0 \mathrm{kcal} / \mathrm{mol}$, and $-12.5 \mathrm{kcal} / \mathrm{mol}$, respectively. Also, a better predicted binding affinity of pediocin PA-1 and salivaricin B against triple mutant was observed compared to the WT. Thus, pediocin PA-1 binds stronger to mutants of the RBD, particularly to double and triple mutants. Salivaricin B showed a better predicted binding affinity towards triple mutant compared to WT, showing that it might be another bacteriocin with potential activity against the SARS-CoV-2 beta variant. Overall, pediocin PA-1, salivaricin P, and salivaricin B are the most promising candidates for inhibiting SARS-CoV-2 (including lineage B.1.351) entrance into the human cells. These bacteriocins derived from lactic acid bacteria hold promising potential for paving an alternative way for treatment and prophylaxis of WT and beta variants.
\end{abstract}

Keywords Beta variant $\cdot$ Bacteriocins $\cdot$ COVID-19 $\cdot$ Protein-protein docking $\cdot$ SARS-CoV-2 $\cdot$ MD simulations

\section{Introduction}

Ismail Erol

ismail.erol@med.bau.edu.tr

Serdar Durdagi

serdar.durdagi@med.bau.edu.tr

Fatih Ortakci

fatih.ortakci@agu.edu.tr

1 Computational Biology and Molecular Simulations Laboratory, Department of Biophysics, School of Medicine, Bahcesehir University, Istanbul, Turkey

2 Department of Biophysics, School of Medicine, Bahcesehir University, Istanbul, Turkey

3 Bioengineering Department, Faculty of Life and Natural Sciences, Abdullah Gul University, Kayseri, Turkey

4 Food Engineering Department, Faculty of Engineering, Erciyes University, Kayseri, Turkey
COVID-19 is caused by a coronavirus named severe acute respiratory syndrome coronavirus 2 (SARS-CoV-2) [1-3]. SARS-CoV-2 has spread globally and devastated public health and economies, with more than 179 million people infected and more than 3.8 million deceased to date (www. worldometers.info). The spike (S) protein, which is responsible for binding to the host receptor and viral entry, consists of S1 and S2 subunits. SARS-CoV-2 infects host cells via binding to angiotensin-converting enzyme II (ACE 2) [4], which is dominantly present on the epithelial cells [5]. Thus, blocking the spike can be considered as an effective approach to prevent SARS-CoV-2 infection. ACE2 is a regulator of gut inflammation which is more expressed in the ileum and colon than in lung $[6,7]$. More than $60 \%$ of patients diagnosed with COVID-19 reported showing diarrhea, nausea, 
and vomiting gastrointestinal symptoms. More importantly, patients having gastrointestinal complaints had overall experienced severe disease outcome [8-10]. Thus, a potential role of gut microbiota and COVID-19 progression and severity could be linked [11-13].

According to International Scientific Association of Probiotics and Prebiotics (ISAPP) the term postbiotics can be described as "preparation of inanimate microorganisms and/ or their components that confers a health benefit on the host" [14]. These could include non-viable bacterial or complex mixture of healthy metabolic products or secreted components like short-chain fatty acids, vitamins, amino acids, organic acid enzymes, secreted proteins, or peptides such as bacteriocins [15-17]. As biotherapeutic agents, bacteriocins or individual cellular components can be directly used to target a particular disease, which may help to provide clear evidence for the effect of the biomolecules. Instead, to study the same with probiotics might yield unclear cause and effect relationship due to dynamic and complex morphology of bacteria. Thus, several bacteriocin molecules draw attention because of their known biochemical structure, storage stability, and the capability to induce mechanisms to control inflammation, adhesion of pathogens to GIT, obesity, hypertension, coronary artery diseases (CVD), cancer, and oxidative stress. And several bacteriocin preparations were patented as bio-therapeutics for a specific health claim, "immune-modulation" [16, 18].

Bacteriocins have recently drawn attention due to the COVID-19 pandemic, which is widespread around the world. Bacteriocins are ribosomally produced peptides with bactericidal effects, known to be produced by several bacteria, including lactic acid bacteria [11]. The antibacterial activities of bacteriocins have been well understood, but the basis of their antiviral activities has been embraced with a lot less attention. Various research groups worldwide probed on their antiviral activities; for example, Todorov et al. (2005) reported that the enterocin CRL35 peptide produced by Enterococcus mundtii ST4V inhibited the replication of Herpes simplex virus (HSV) type 1 and 2 [19]. Ferir et al. (2010) reported that Labyrinthopeptin A1, a carbacyclic lantibiotic of Actinomadura namibiensis DSM 6313, showed antiviral activity against HIV-1 and HSV-1 [20]. Furthermore, Serkedjieva et al. (2000) showed that the replication of H7N7 and H7N1 strains of influenza virus was highly specifically inhibited by $5.0-\mathrm{kDa}$ bacteriocin of Lactobacillus delbrueckii subsp. bulgaricus in chicken embryo fibroblast [21]. Quintana et al. (2014) reported that bacteriocins of Lactococcus lactis GLc03 and GLc05 showed virucidal effect on HSV-1 and poliovirus (PV-1) [22]. Recently, Anwar et al. (2020) studied the molecular dynamics (MD) simulations of plantaricin peptides (W, JLA-9, D, and BN) against spike proteins (S), human-ACE-2, and RNA-dependent RNA polymerase $(\mathrm{RdRp})$ [23]. The results of their study revealed that plantaricin peptides (W, JLA-9, D, and BN) blocked the entry into the cell by binding to $S$ protein, ACE-2, and RdRp. In another study, Manna et al. (2020) demonstrated a higher binding tendency of subtilisin, curvacin A, sakacin $\mathrm{P}$, lactococcin $\mathrm{Gb}$ to S-protein of SARS-CoV-2, and human ACE-2 by using in silico approach [24]. To our knowledge, no bacteriocin activity against the SARS-Cov- 2 beta variant (lineage B.1.351) has been reported so far. Therefore, elucidation of the bacteriocin-S protein interactions at the atomic level is critical to fill this gap in the literature. Thus, the present study focused on the potential therapeutic functions of several bacteriocins against SARS-CoV-2. We utilized an in silico protein-protein docking approach to elucidate the binding affinity of bacteriocin peptides to the RBD of SARS-CoV-2. Ultimately, the role of lactic acid bacteriaderived bacteriocins on strengthening the immune defenses against fighting coronaviruses of wild type and beta variant could be uncovered.

\section{Materials and Methods}

\section{In Silico Preparation of Antimicrobial Peptides and Receptors}

We collected 22 bacteriocin sequences from PubChem. MarvinSketch was used to draw and convert to 3D structures of the peptides (MarvinSketch 19.13, ChemAxon; https://www.chemaxon. com, Budapest, Hungary). We considered uncharged $\mathrm{N}$ - and C-terminal ends in our docking calculations. (In our dataset, most of the C-terminals have- $\mathrm{NH} 2$ and-H ending; this option led us to consider only carbonyl group the $\mathrm{C}$-term). Folded structures of the peptides were predicted using the PEP-FOLD3 algorithm [25]. Then obtained folded structures were incorporated into Schrodinger's Maestro suite (Schrodinger LLC, New York, NY) [26, 27]. PHE14 of Enterocin EJ97 (139586139) and CYS1 of Gassericin B2 (139586609) were methylated in the original structure, and we deleted methyl moiety from both sequences. Coronavirus RBD-ACE2 (PDB ID: 6LZG) complex was downloaded from Protein Data Bank. All solvent molecules and ACE2 were deleted. Protein preparation wizard was used to predict protonation states of the side chains at $\mathrm{pH} 7.4$ of both peptides and RBD [28, 29]. All beta variants (K417N, K417NE484K, and K417N-E484K-N501Y mutants) RBDs were generated using Maestro (Schrodinger LLC).

\section{Protein-Protein Docking Simulations}

HADDOCK v2.4 was used in protein-protein docking simulations [30]. Active residues at the binding pocket of RBD were obtained from the literature [31, 32]. Following residues were defined as active: LYS417, GLY446, TYR449, TYR453, LEU455, PHE456, ALA475, GLY476, GLU484, 
PHE486, ASN487, TYR489, PHE490, GLN493, GLY496, GLN498, THR500, ASN501, GLY502, and TYR505 at the RBD. The default protocol with default parameter sets for docking and refinement was used on the server. Passive residues were automatically defined around the active residues. Top complexes from HADDOCK were downloaded and submitted to the PROtein binDIng enerGY prediction (PRODIGY) server, and binding energies were obtained [33]. Protein-protein interfaces were evaluated with GetContacts scripts (https://getcontacts.github. io/). All figures were generated with PyMol v2.1 (https:// github.com/schrodinger/pymol-open-source (Schrodinger LLC, New York, NY)) [34]. Throughout the manuscript, we denoted bacteriocins as chain $\mathrm{A}$ and the RBD as chain B. For instance, A8-B453 means the interaction between Bacteriocins 8th amino acid and RBDs 453rd amino acid.

\section{Molecular Dynamics Simulation}

Among all docking results, the best performing ligand was pediocin PA-1 (56842033) against WT and mutant RBDs. We simulated complexes of WT, K417N, K417N_E484K, and K417N_E484K_N501Y with pediocin PA-1 for 500 ns and as three independent runs. In total, we have simulated $6 \mu$ s for all simulation systems (such as WT- pediocin PA-1 $500 \mathrm{~ns} \times 3$ times, K417N-pediocin PA-1 $500 \mathrm{~ns} \times 3$ times). Desmond MD code (D. E. Shaw Research, New York, NY) was used to simulate systems with Martyna Tobias Klein (MTK) [35] barostat to control pressure at $1.01 \mathrm{bar}$, and with Nosé-Hoover $[36,37]$ thermostat to control the temperature at $310 \mathrm{~K}$. OPLS3 force field was used in all simulations [38]. Reversible reference system propagator algorithms (RESPA) were used to calculate bonded and non-bonded interactions. Protein-Ligand systems were solvated in the TIP3P water model [39] and in an orthorhombic box with a 10- $\AA$ buffer distance in all $x, y$, and $z$ dimensions. The $0.15 \mathrm{M} \mathrm{NaCl}$ ionic salt concentration was used to neutralize the medium. Each system was simulated for $500 \mathrm{~ns}$, and a total of 5000 frames was collected and saved. Two-hundred frames out of 5000 trajectory frames were used in Molecular Mechanics Generalized Born Surface Area (MM-GBSA) calculations. The Prime module of Schrodinger's Maestro package (Schrodinger LLC) [40, 41] was used to calculate relative binding free energies throughout the simulation along with the VSGB 2.0 [42] implicit water model.

\section{Hydrogen Bond Analysis and Trajectory Clustering}

The number of hydrogen bonds between pediocin PA- 1 and RDBs was calculated using the Maestro package (Schrodinger LLC). The resultant hydrogen bond numbers for each system were converted to a probability distribution. For smoothing, "cubic splines" with a smoothing parameter of
0.99449 was used in MATLAB 2019b curve fitting toolbox (The MathWorks, Inc., Natick, MA). Before concatenation of the trajectories, all ions and water molecules were stripped out. All frames opened and aligned to starting structure in Visual Molecular Dynamics (VMD) [43]. Aligned trajectories were saved in GROMACS trr format, and gmx trjcat was used to concatenate three independent runs [44]. After concatenation, we obtained a single 15,000 frame long single trajectory for each system. Gromacs clustering algorithm was applied with the $3-\AA$ cut-off using the gmx cluster tool [45]. The following clusters were obtained, 23, 19, 30, and 18 , for the WT, K417N-RBD, K417N-E484K-RBD, and K417N-E484K-N501 Y-RBD, respectively. The first member of each cluster was used to evaluate interactions at the interface and plotting figures.

\section{Statistical Analysis}

MM/GBSA scores of WT and mutants are treated as independent samples ( 3 replicates of 200 frames). Each frame is separated by $2.5 \mathrm{~ns}$. Since distributions are not necessarily normally distributed, we used the Wilcoxon signed-rank test on datasets. MATLAB 2019b (The MathWorks, Inc., Natick, MA) statistical toolbox was used.

\section{Results}

\section{Molecular Docking Simulations Reveal Pediocin PA-1 as the Tightest Binder}

In all WT and mutants, pediocin PA-1 was the tightest binder. Salivaricin P comes second for the WT and K417N. For the K417N-E484K double and K417N-E484K-N501Y triple mutants, salivaricin B achieved second best score, where salivaricin $\mathrm{P}$ took third place. Among all used peptides, pediocin PA-1, salivaricin P, and salivaricin B were the best three according to docking scores. Plantaricin JLA-9 was found the weakest binder among all. Interestingly, pediocin PA-1 binding energies get even lower upon the mutations, and tighter complexes are observed. In K417N-E484K double mutant, pediocin PA-1 binding was found the lowest energy complex compared to all other mutant-peptide complexes (Table 1). Pediocin PA-1 binding poses at the interface of WT and mutants represented in Fig. 1 and Figure S1. Table 2 shows the interactions between pediocin PA- 1 and RBD. A3-B492, A12-B416, and A39-B484 interactions were only observed in WT docking poses. A2-B446 interaction was the only interaction that was observed solely in the K417N mutant. A2-B444, A3-B449, A3-B493, A4-B498, and A21-B505 were only observed in K417N-E484K double mutant. A5-B449, A6-B449, A6-B501, A7-B505, and A20-B505 interactions were merely observed in the 
Table 1 Protein-protein docking results of studied bacteriocins $(\mathrm{kcal} / \mathrm{mol})$

\begin{tabular}{|c|c|c|c|c|c|}
\hline Peptides & PubChem ID & WT & $\mathrm{K} 417 \mathrm{~N}$ & K417N_E484K & $\begin{array}{l}\mathrm{K} 417 \mathrm{~N}_{-} \\
\mathrm{E} 484 \mathrm{~K} \\
\mathrm{~N} 501 \mathrm{Y}\end{array}$ \\
\hline Pediocin PA-1 & 56842033 & -12.0 & -12.3 & -13.8 & -13.6 \\
\hline Salivaricin $\mathrm{P}$ & 139587600 & -12.0 & -11.3 & -12.5 & -11.6 \\
\hline Enterocin EJ97 & 139586139 & -10.8 & -10.2 & -10.7 & -10.6 \\
\hline Lactococcin Mmfii & 139588229 & -10.5 & -10.5 & -11.1 & -10.7 \\
\hline Gassericin B2 & 139586609 & -10.4 & -8.9 & -9.7 & -10.0 \\
\hline Carnobacteriocin & 139583933 & -10.4 & -10.6 & -11.0 & -10.9 \\
\hline Plantaricin GZ1-27 & 146684209 & -10.3 & -10.1 & -10.3 & -10.5 \\
\hline Gassericin B1 & 139586327 & -10.3 & -9.4 & -9.6 & -9.7 \\
\hline Plantaricin D & 139586697 & -10.2 & -9.3 & -8.7 & -10.3 \\
\hline Bavaricin A & 139588080 & -10.0 & -9.6 & -9.7 & -9.5 \\
\hline Plantaricin W & 139586,573 & -10.0 & -10.6 & -11.5 & -10.1 \\
\hline Gassericin B3 & 139586277 & -9.6 & -9.7 & -9.8 & -9.5 \\
\hline Caseicin B & 16730443 & -9.6 & -9.2 & -9.1 & -9.1 \\
\hline Salivaricin B & 16198259 & -9.6 & -10.1 & -13.0 & -12.3 \\
\hline Leucocin B-TA33a & 139584476 & -9.4 & -10.6 & -9.3 & -9.5 \\
\hline Leucocin B-TA11A & 139587056 & -9.1 & -10.1 & -10.0 & -10.8 \\
\hline Enterocin A & 139587,481 & -9.0 & -10.6 & -9.9 & -10.3 \\
\hline Enterocin CRL 35 & 139586357 & -9.0 & -9.8 & -9.5 & -9.9 \\
\hline Caseicin A & 101561458 & -9.0 & -9.9 & -9.5 & -9.6 \\
\hline Leucocin C-TA33a & 139583454 & -8.6 & -10.9 & -10.5 & -10.9 \\
\hline Mutacin & 139588082 & -8.3 & -10.0 & -9.0 & -8.7 \\
\hline Plantaricin JLA-9 & 132535900 & -7.9 & -8.9 & -8.6 & -8.1 \\
\hline
\end{tabular}

K417N-E484K-N501Y triple mutant. A8-B453, A11-B473, A15-B403, and A43-B484 interactions were observed in all docking poses. Interestingly, A43 of the pediocin PA-1 interacted with residue number 484 of RBDs in all studied WT and mutant systems.

In salivaricin $\mathrm{P}$ docking poses, the following interactions are given in Table S1. Salivaricin P binding poses at the interface of WT and mutants given in Figure S2. A2-B449, A10-B498, A10-B501, A10-B496, A15-B487, and A18B489 interactions were observed for only the WT-salivaricin P complex. The K417N mutant, A3-B493, A6-B492, A6-B494, A9-B494, A10-B493, A13-B489, and A17-B489 interactions were not observed in all other systems, and we can conclude that these interactions were $\mathrm{K} 417 \mathrm{~N}$ specific interactions. A1-B416, A1-B421, A1-B420, A4-B417, A7-B421, A9-B487, A10-B489, A10-B487, A11-B493, A11B490, A14-B496, A16-B498, and A18-B505 interactions were only observed in K417N-E484K double mutant. For the K417N-E484K-N501 Y triple mutant, A1-B488, A1-B485, A4-B487, A7-B492, A9-B501, A9-B498, A9-B446, A11B406, A11-B403, A12-B453, A13-B403, and A15-B421 interactions were detected, and these interactions were not observed in all other docking poses. We could not be able to find any common interaction for salivaricin P and RBD systems.
Table S2 shows enterocin EJ97-RDB interactions. Figure S3 depicts enterocin EJ97 at the binding interface of WT and mutants. A9-B495, A13-B498, A13-B500, and A15-B498 interactions were WT specific interactions and were not observed in all other studied systems. For the K417N mutant, A9-B505 interaction was observed at the binding cavity, which was not seen in all other studied systems. A2-B494, A2-B493, and A12-B498 interactions were K417N-E484K double-mutant specific interactions. For the K417N-E484K-N501Y triple mutant, A4-B489 and A11B501 interactions were detected and found to be case-specific interactions.

\section{Simulation Trajectories Identify K417N-E484K-N501Y Triple-Mutant RBD as the Most Stable System}

Herein, WT-pediocin PA-1 simulations, we observed RMSD averages as $2.20 \pm 0.35,1.97 \pm 0.26$, and $2.21 \pm 0.24 \AA$. In all simulations, RMSD values of RBDs converged just after $50 \mathrm{~ns}$. Figure S4 shows the WT-RBD root mean square deviation (RMSD) graph. The average RMSD of the backbone atoms of the WT-RBD simulations was found as $2.13 \AA$ (the average of the three runs). Very similar RMSD graphs were observed in the case of K417N-pediocin PA-1 complexes compared to WT. 


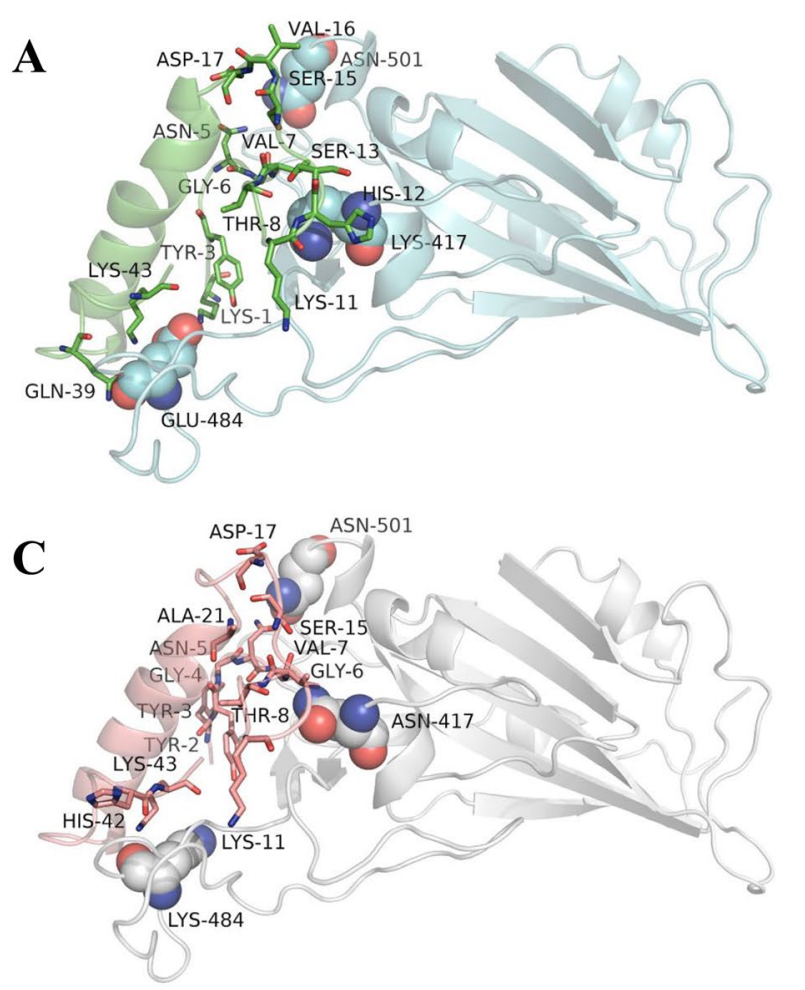

Fig. 1 In silico analysis of the binding interactions between predicted Pediocin PA-1 peptide and different mutants of the spike protein complex. (A) WT-RBD-Pediocin PA-1, (B) K417N-RBD-Pediocin PA-1, (C) K417N-E484K-RBD-Pediocin PA-1, and (D) K417N-E484K-

RMSD averages were calculated $2.15 \pm 0.24,2.23 \pm 0.26$, and $1.97 \pm 0.13 \AA$, respectively (see Figure S5). The average of the RMSD for the K417N mutant backbone atoms was calculated as $2.11 \AA$ (the average of the three runs). For the K417N-E484K double-mutant-pediocin PA-1 simulations, we found backbone RMSD averages $2.38 \pm 0.41,2.15 \pm 0.33$, and $2.44 \pm 0.40 \AA$, respectively (Figure S6). The average of the three runs was $2.32 \AA$ (the average of the three runs). K417N-E484K-N501Y triple mutant has the lowest average backbone RMSD value of $2.05 \AA$ A compared to other systems (the average of the three runs). For the three replicates, the following averages were found: $1.91 \pm 0.28,2.04 \pm 0.19$, and $2.21 \pm 0.50 \AA$, respectively (see Figure S7). When the backbone RMSD values were compared in all simulations, very similar behavior was observed. RBDs reached the plateau a very short time (around $\sim 0 \mathrm{~ns}$ ) after the start of simulation in both the WT and mutant states. The lowest RMSD average was observed in the K417N-E484K-N501Y triple mutant, while the highest RMSD average was observed for the K417N-E484K double mutant.

\section{The Most Stable Conformation of Pediocin PA-1 Observed in K417N}

K417N mutant has the lowest RMSD average for the pediocin PA- 1 compared to others and is found to be $4.07 \AA$ (the
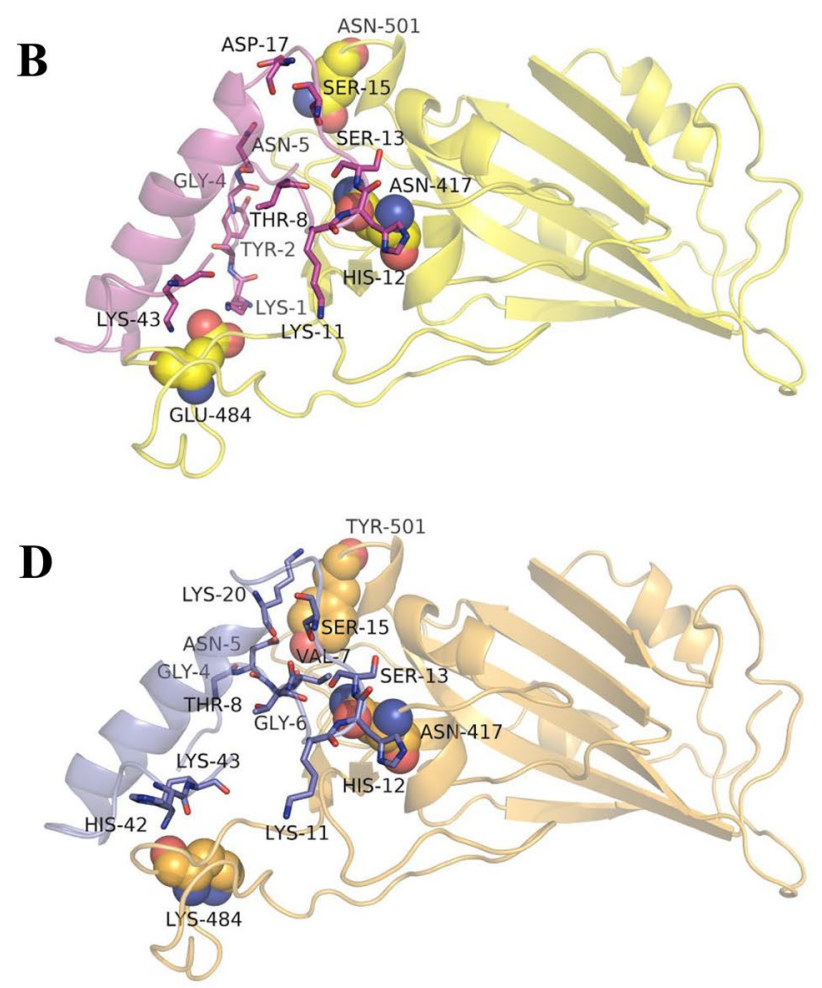

N501Y-RBD-Pediocin PA-1. Figures were prepared using the best docking pose (simulation starting structure). Pediocin PA-1 residues interacting with RBD are shown in stick and beta variant mutations shown in spheres

average of the three runs). The second lowest average RMSD value was found in the K417N-E484K-N501Y triple mutant as $4.44 \AA$ (the average of the three runs). For the K417N-E484K double mutant and WT, RMSD values of pediocin PA-1 backbone atoms were found at 4.49 and $5.00 \AA$, respectively (the average of the three runs). Figure S13 shows the backbone RMSD values of all studied systems. Figure S14 depicts backbone RMSD values for the pediocin PA-1 at the WT-RBD complexes. RMSD averages $4.19 \pm 0.55 \AA, 6.13 \pm 1.10 \AA$, and $4.68 \pm 0.69 \AA$ were obtained for the repeats, respectively. In the second run, we observed a different profile for the backbone atom RMSD of the pediocin PA-1. The backbone RMSD of the pediocin PA-1 at the K417N-RBD complexes was given in Figure $S 15$. We observed $3.30 \pm 0.93 \AA, 4.12 \pm 0.48 \AA$, and $4.80 \pm 0.96 \AA$ for the repeats, respectively. Run- 2 and run- 3 were not changed too much after $100 \mathrm{~ns}$ (see Figure S15). In K417N-E484K double-mutant RBD and pediocin PA-1 complexes, for the run-1, run-2, and run-3, we observed $5.61 \pm 0.79 \AA, 3.38 \pm 0.38 \AA$, and $4.47 \pm 0.92 \AA$, respectively (see Figure S16). For the K417N-E484K-N501Y triple-mutant RBD-pediocin PA-1 simulation repeats, we observed a very similar RMSD profile for the run-1 and run-2, especially in the last $250 \mathrm{~ns}$. The averages are given in Figure S17, and for the simulation, repeats were 
Table 2 Pediocin PA-1-Spike Protein RBD domain interactions. Pediocin PA-1 presented as chain A and the RBD as chain B. In A:1, A represents chain $A$ and number (in this case, 1) after the colon depicts the residue number. 1 (bold) means interaction observed, and 0 (italics) means no interaction. Data created using simulation starting structures

\begin{tabular}{|c|c|c|c|c|c|}
\hline Pediocin PA-1 & RBD & WT & K417N & $\begin{array}{l}\mathrm{K} 417 N_{-} \\
\mathrm{E} 484 \mathrm{~K}\end{array}$ & $\begin{array}{l}\mathrm{K} 417 \mathrm{~N}_{-} \\
\mathrm{E} 484 \mathrm{~K} \\
\mathrm{~N} 501 \mathrm{Y}\end{array}$ \\
\hline $\mathrm{A}: 1$ & $B: 484$ & 1 & 1 & 0 & 0 \\
\hline $\mathrm{A}: 2$ & B:444 & 0 & 0 & 1 & 0 \\
\hline $\mathrm{A}: 2$ & $B: 446$ & 0 & 1 & 0 & 0 \\
\hline $\mathrm{A}: 3$ & B:449 & 0 & 0 & 1 & 0 \\
\hline $\mathrm{A}: 3$ & B:493 & 0 & 0 & 1 & 0 \\
\hline $\mathrm{A}: 3$ & B:492 & 1 & 0 & 0 & 0 \\
\hline A:4 & B:498 & 0 & 0 & 1 & 0 \\
\hline A:4 & B:449 & 0 & 1 & 1 & 1 \\
\hline A:5 & B:449 & 0 & 0 & 0 & 1 \\
\hline A:5 & B:501 & 0 & 0 & 1 & 1 \\
\hline A:5 & B:498 & 1 & 1 & 0 & 0 \\
\hline A:6 & B:449 & 0 & 0 & 0 & 1 \\
\hline A: 6 & B:501 & 0 & 0 & 0 & 1 \\
\hline A: 6 & B:496 & 1 & 0 & 1 & 1 \\
\hline A:7 & B:505 & 0 & 0 & 0 & 1 \\
\hline A:7 & B:453 & 1 & 0 & 1 & 0 \\
\hline $\mathrm{A}: 8$ & B:453 & 1 & 1 & 1 & 1 \\
\hline $\mathrm{A}: 11$ & B:473 & 1 & 1 & 1 & 1 \\
\hline A:11 & B:457 & 1 & 1 & 0 & 0 \\
\hline A:12 & B:421 & 0 & 1 & 0 & 1 \\
\hline A:12 & B:417 & 0 & 1 & 0 & 0 \\
\hline A:12 & B:420 & 1 & 1 & 0 & 1 \\
\hline $\mathrm{A}: 12$ & B:416 & 1 & 0 & 0 & 0 \\
\hline $\mathrm{A}: 13$ & B:417 & 1 & 1 & 0 & 1 \\
\hline A:15 & B:505 & 0 & 1 & 1 & 1 \\
\hline A:15 & B:403 & 1 & 1 & 1 & 1 \\
\hline A:16 & B:505 & 1 & 0 & 0 & 0 \\
\hline A: 17 & B:505 & 1 & 1 & 1 & 0 \\
\hline$A: 20$ & B:505 & 0 & 0 & 0 & 1 \\
\hline $\mathrm{A}: 21$ & B:505 & 0 & 0 & 1 & 0 \\
\hline A:39 & B:484 & 1 & 0 & 0 & 0 \\
\hline A:42 & B:489 & 0 & 0 & 1 & 1 \\
\hline A:43 & B:490 & 0 & 0 & 1 & 1 \\
\hline $\mathrm{A}: 43$ & B:484 & 1 & 1 & 1 & 1 \\
\hline
\end{tabular}

found as $5.13 \pm 0.62 \AA, 4.80 \pm 0.73 \AA$, and $3.38 \pm 0.52 \AA$, respectively.

\section{The Lowest MM/GBSA Binding Energy Observed in K417N-Pediocin PA-1 System}

All MM/GBSA binding free energies for all studied systems are given in Figure S8. Figures S9-S12 depict the system-wise MM/GBSA energies for the WT, K417N, K417N-E484K, and K417N-E484K-N501Y mutants. The averages for the studied systems calculated were as follows: $-101.50,-132.68,-109.63$, and $-129.26 \mathrm{kcal} /$ mol, respectively. The lowest MM/GBSA binding energy was observed in the K417N system (see Figure S10). However, the highest score corresponds to WT (see Figure S9). Similar to docking results, in MM/GBSA energies, WT was the highest one compared to all others. Based on the MM/GBSA binding energies, we conclude that the predicted affinity of pediocin PA-1 increases upon mutation. Box plots of the MM/GBSA binding energy distributions are given in Fig. 3A. All mutants are significantly different from the WT. Two distributions that did not reject the null hypothesis were K417N and K417N-E484K-N501Y.

\section{Simulation Cluster Structures Show Different Interaction Patterns}

When compared the starting structure and cluster structure interactions at the interface, we observed that the A1-B484 interaction was conserved in both WT and K417N. However, the A3-B493 interaction was conserved in $\mathrm{K} 417 \mathrm{~N}$ E484K double mutant and newly established for the WT and K417N. Although A5-B498 interaction was preserved for the WT, it was lost in the K417N mutation. The same interaction occurred in the K417N-E484K double mutant. A43-B484 interaction was conserved only in $\mathrm{K} 417 \mathrm{~N}$ and K417N-E484K mutations observed across all simulation starting structures. A17-B505 and A20-B505 interactions were conserved only in K417N and K417N-E484K mutations, respectively (see Table 2 and 3).

In Fig. 2 and Figure S22, we plotted the first member of each cluster. First members of each cluster cover 30.19\%, $30.37 \%, 28.32 \%$, and $28.00 \%$ of total frames for the WT, K417N, K417N-E484K, and K417N-E484K-N501Y, respectively. Polar interactions between $\mathrm{RBD}$ and pediocin PA-1 are given as dashes (see Fig. 2). A1-B484, A3-B484, A3-B493, A4-B494, A5-B493, A5-B498, A6-B453, A27B498, A35-B484, and A39-B493 were observed between WT-RBD and pediocin PA-1 (Fig. 2A). For the K417NRBD-pediocin PA-1 complex, the following polar interactions were detected in the cluster structure: A1-B484, A3-B484, A3-B493, A4-B446, A5-B501, A6-B496, A17B403, A17-B505, A41-B487, and A42-B485 (Fig. 2B). The A3-B493, A4-B494, A5-B403, A5-B453, A5-B496, A5-B498, A11-B415, A11-B420, A20-B405, and A20-B406 polar interactions were found in K417N-E484K-RBD and pediocin PA-1 cluster structure (Fig. 2C). The A3-B494, A5-B494, A9-B417, A11-B415, A11-B417, A11-B420, A11-B460, A20-B505, A40-B486, and A43-B484 polar interactions were found in the K417N-E484K-N501Y triplemutant and pediocin PA-1 cluster structure (Fig. 2D). 
Table 3 Pediocin PA-1-Spike Protein RBD domain interactions. Pediocin PA-1 presented as chain A and the RBD as chain B. In A:1, $A$ represents chain $A$ and number (in this case, 1) after the colon depicts the residue number. 1 (bold) means interaction observed, 0 (italics) means no interaction. Data was created using cluster structures

\begin{tabular}{|c|c|c|c|c|c|}
\hline Pediocin PA-1 & RBD & WT & $\mathrm{K} 417 \mathrm{~N}$ & $\begin{array}{l}\mathrm{K} 417 N_{-} \\
\mathrm{E} 484 \mathrm{~K}\end{array}$ & $\begin{array}{l}\mathrm{K} 417 \mathrm{~N} \\
\mathrm{E} 484 \mathrm{~K} \\
\mathrm{~N} 501 \mathrm{Y}\end{array}$ \\
\hline A:1 & B:484 & 1 & 1 & 0 & 0 \\
\hline $\mathrm{A}: 3$ & $\mathrm{~B}: 484$ & 1 & 0 & 0 & 0 \\
\hline A:3 & B:493 & 1 & 1 & 1 & 0 \\
\hline $\mathrm{A}: 3$ & B:494 & 0 & 0 & 1 & 1 \\
\hline A:4 & B:446 & 0 & 1 & 0 & 0 \\
\hline A:4 & B:492 & 0 & 0 & 0 & 1 \\
\hline $\mathrm{A}: 4$ & B:493 & 1 & 0 & 0 & 0 \\
\hline $\mathrm{A}: 4$ & B:494 & 1 & 0 & 1 & 0 \\
\hline A:4 & B:496 & 0 & 0 & 1 & 0 \\
\hline$A: 5$ & B:403 & 0 & 0 & 1 & 0 \\
\hline $\mathrm{A}: 5$ & $\mathrm{~B}: 453$ & 0 & 0 & 1 & 0 \\
\hline A:5 & B:493 & 1 & 0 & 0 & 1 \\
\hline A:5 & B:496 & 0 & 0 & 1 & 0 \\
\hline$A: 5$ & B:498 & 1 & 0 & 1 & 0 \\
\hline A:5 & B:501 & 0 & 1 & 0 & 0 \\
\hline A:6 & B:453 & 1 & 0 & 0 & 0 \\
\hline A:6 & B:493 & 0 & 0 & 1 & 0 \\
\hline A:6 & $B: 496$ & 0 & 1 & 0 & 0 \\
\hline $\mathrm{A}: 9$ & $\mathrm{~B}: 417$ & 0 & 0 & 0 & 1 \\
\hline $\mathrm{A}: 11$ & B:415 & 0 & 0 & 1 & 1 \\
\hline $\mathrm{A}: 11$ & $\mathrm{~B}: 417$ & 0 & 0 & 0 & 1 \\
\hline $\mathrm{A}: 11$ & B:420 & 0 & 0 & 1 & 1 \\
\hline $\mathrm{A}: 11$ & $B: 460$ & 0 & 0 & 0 & 1 \\
\hline $\mathrm{A}: 12$ & B:409 & 0 & 0 & 0 & 1 \\
\hline $\mathrm{A}: 12$ & B:417 & 0 & 0 & 0 & 1 \\
\hline $\mathrm{A}: 17$ & B:403 & 0 & 1 & 0 & 0 \\
\hline $\mathrm{A}: 17$ & B:505 & 0 & 1 & 0 & 0 \\
\hline A:20 & B:405 & 0 & 0 & 1 & 0 \\
\hline A:20 & B:406 & 0 & 0 & 1 & 0 \\
\hline $\mathrm{A}: 20$ & $B: 505$ & 0 & 0 & 0 & 1 \\
\hline $\mathrm{A}: 27$ & B:498 & 1 & 0 & 0 & 0 \\
\hline $\mathrm{A}: 35$ & B:484 & 1 & 0 & 0 & 0 \\
\hline $\mathrm{A}: 38$ & B:477 & 0 & 0 & 0 & 1 \\
\hline A:38 & B:478 & 0 & 0 & 0 & 1 \\
\hline A:38 & B:487 & 0 & 0 & 0 & 1 \\
\hline A:38 & B:490 & 1 & 0 & 0 & 0 \\
\hline $\mathrm{A}: 39$ & B:493 & 1 & 0 & 0 & 0 \\
\hline $\mathrm{A}: 40$ & B:486 & 0 & 0 & 0 & 1 \\
\hline $\mathrm{A}: 41$ & $\mathrm{~B}: 487$ & 0 & 1 & 0 & 0 \\
\hline $\mathrm{A}: 42$ & B:485 & 0 & 1 & 0 & 0 \\
\hline $\mathrm{A}: 43$ & B:484 & 0 & 1 & 0 & 1 \\
\hline
\end{tabular}

TYR2, TYR3, THR8, LYS20, HIS38, and HIS42 of Pediocin PA-1 Identified as Important Residues

A3-B492 interaction was observed in docking pose; however, this contact was lost in MD simulations. Interestingly, this interaction was shifted to A3-B493 and conserved as $84 \%, 15 \%$, and $30 \%$ in WT simulations (Table S3). A12B416 interaction was detected in docking pose; in MD simulations, it was completely lost. The A39-B484 interaction was WT specific, which was conserved at $12 \%, 9 \%$, and $40 \%$ throughout the MD trajectories (Table S3). A2-B446 interaction was the only interaction observed in $\mathrm{K} 417 \mathrm{~N}$ mutant compared to WT, double, and triple mutants. However, during MD simulations, this interaction was conserved at 63\% and $15 \%$ in $\mathrm{K} 417 \mathrm{~N}-1$ and $\mathrm{K} 417 \mathrm{~N}-3$ replicas (Table S3). In the second run, this contact was completely lost. On the other hand, a new interaction was formed between A2-B449 and conserved at $38 \%, 6 \%$, and $61 \%$ during $\mathrm{MD}$ simulations (Table S3). In the case of the $\mathrm{K} 417 \mathrm{~N}-484 \mathrm{~K}$ double-mutant system, A2-B444, A3-B449, and A21-B505 interactions were determined as specific contacts. However, none of these contacts were conserved throughout the MD simulations. A3-B493 interaction was observed as $91 \%, 57 \%$, and $6 \%$ during triplicate simulations (Table S3). However, the A4-B498 interaction was again disrupted, and the percentage of this interaction decreased to $6 \%$ and $7 \%$ in the second and third simulations, respectively (Table S3). A5-B449, A6-B449, A6-B501, and A7-B505 interactions were specific to triple mutant. However, none of these interactions were conserved during MD simulations. The A20-B505 interaction was the only interaction conserved at $1 \%, 18 \%$, and $8 \%$ and was triple mutant specific (Table S3). A2-B449, A3-B493, A3-B494, A8-B455, A8-B493, A20-B505, A38B486, A42-B486, and A42-B489 interactions were similar across all replicas and systems. Therefore, we can conclude that TYR2, TYR3, THR8, LYS20, HIS38, and HIS42 were important residues for pediocin PA-1. Crucial RBD residues were TYR449, LEU455, PHE486, TYR489, GLN493, SER494, and ASN-TYR505.

\section{The Highest Number of Hydrogen Bonds Observed in K417N Mutant}

The H-bond probability distributions are plotted in Fig. 3B. Observed average number of hydrogen bonds were $8 \pm 2$, $6 \pm 3$, and $10 \pm 3$ for the WT-pediocin PA- 1 complexes in 3 repeats, respectively. Figure S18 depicts the H-bonding pattern throughout the MD repeats. We observed similar patterns in the run- 1 and run-2 in the last $200 \mathrm{~ns}$ of the simulations. The highest number of H-bonds was observed in run-3 for the WT-pediocin PA-1 complexes (see Figure S18). K417N-pediocin PA-1 H bonding patterns are given in Figure S19. After the first 100 ns, we observed very 


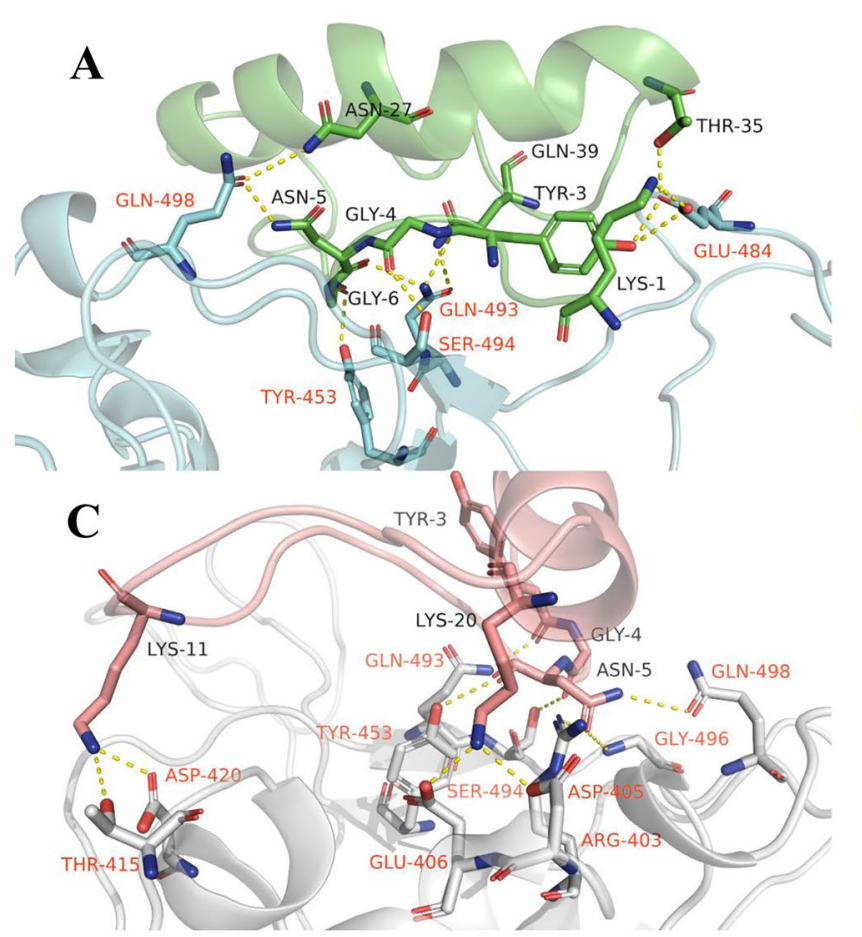

Fig. 2 In silico analysis of the binding interactions between predicted pediocin PA-1 peptide and different mutants of the spike protein complex. (A) RBD_WT-pediocin PA-1, (B) RBD_K417N-pediocin PA-1, (C) RBD_K417N_E484K-pediocin PA-1, and (D) RBD_K417N_

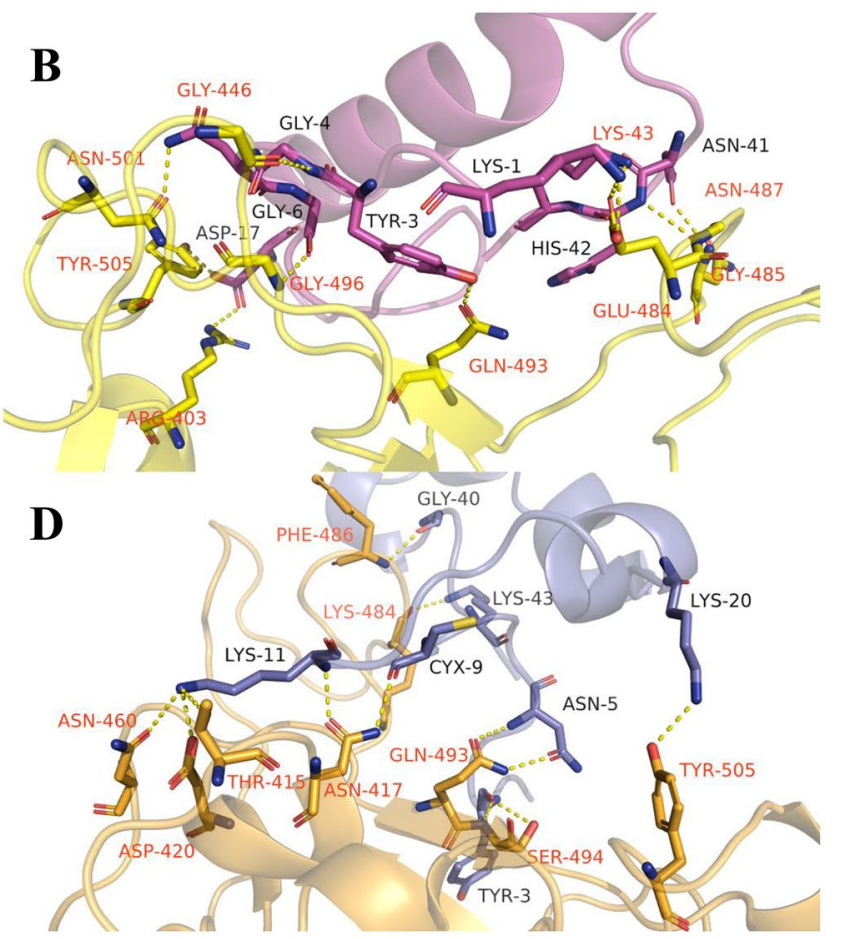

E484K_N501Y-pediocin PA-1. Figures were prepared using cluster structures. Polar interactions were plotted, and the interacting residues were labeled. RBD residues labeled in red, pediocin PA-1 residues colored in black

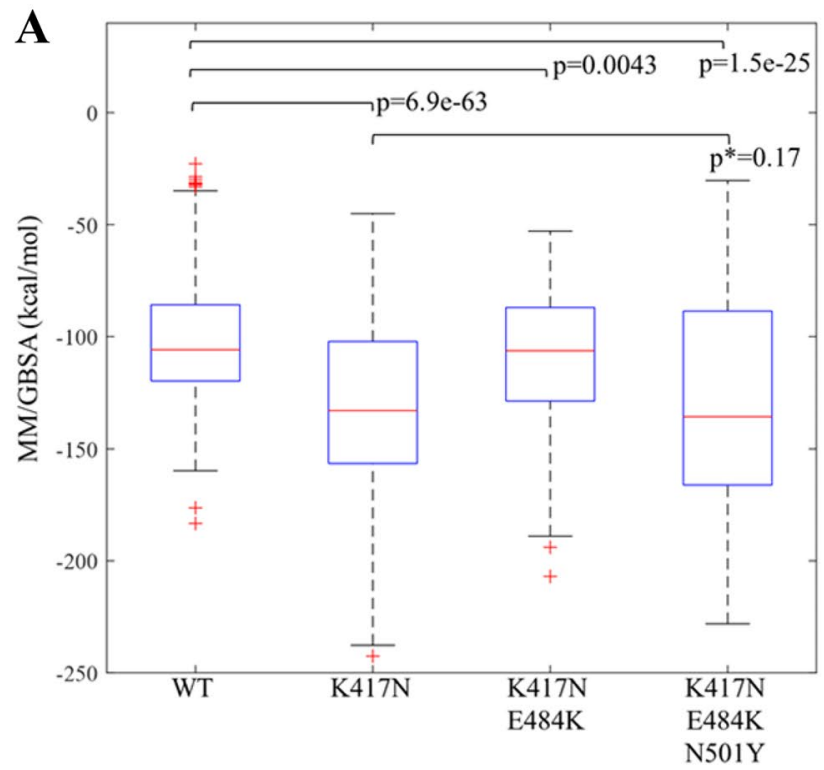

Fig. 3 (A) Box plot representation of the MM/GBSA scores. All averages are significantly different from each other. However, the averages of K417N-RBD and K417N-E484K-N501Y-RBD averages

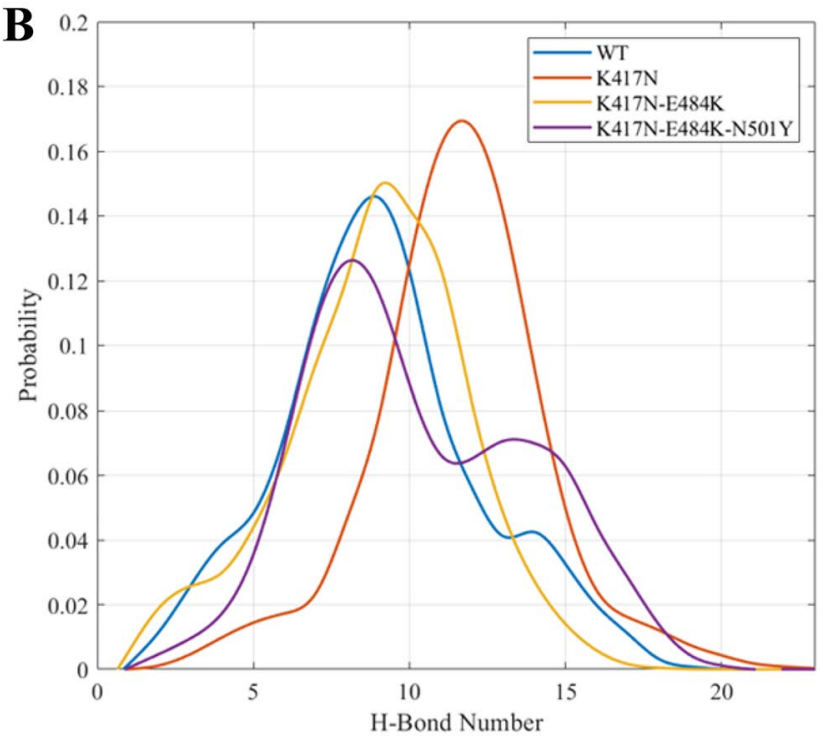

are not significantly different. (B) Probability distributions of H-bond numbers in each system. K417N-RBD system differs from others slightly 
similar binding patterns in all repeats. The averages for the repeats were $11 \pm 3,10 \pm 3$, and $11 \pm 2$, respectively. Also, in the last $80 \mathrm{~ns}$, a decrease was observed in run-1, which differentiated slightly from run-2 and run-3 (see Figure S19). For the K417N-E484K double-mutant and pediocin PA-1 complexes, we observed $10 \pm 2,7 \pm 3$, and $8 \pm 2$ number of H-bonds, respectively. In the last $200 \mathrm{~ns}$, the bonding pattern across the repeats changed (see Figure S20). Run-1 was the simulation with the highest average H-bond numbers compared to other repeats in double-mutant simulations. The H-bonding patterns for the K417N-E484K-N501Y triplemutant RBD and pediocin PA-1 complexes were depicted in Figure S21. The average and the standard deviations for the runs were found $7 \pm 2,13 \pm 2$, and $8 \pm 2$, respectively. In the first $150 \mathrm{~ns}$ of the trajectories, all repeats had a different $\mathrm{H}$ bonding pattern. However, after $200 \mathrm{~ns}$, run- 1 and run- 3 converged to a similar binding pattern, and the run-2 differentiated from the other two having the highest number of H-bonds (see Figure S21). Based on H-bond analysis, the most increased number bonding was observed in K417N system, which agrees with MM/GBSA binding energies.

\section{Discussion}

The current pandemic of SARS-CoV-2 has been globally threatening public health [18]. Millions of people have been infected by the coronavirus, and many of them have lost their lives. Therefore, there is an urgent need for a cure for the prevention and treatment of COVID-19 [43]. Even though recently developed vaccines pave the way for prevention, there are still no specific drugs to treat COVID-19. For this urgent need for new drugs, the scientific community has been spending enormous effort to find drug molecules that inhibit the entry of SARS-CoV-2. This could be through the inhibition of key enzymes involved in its life cycle (antiviral agents) or regulation of the cytokine storm in the host (immunomodulators) [44, 45]. Many synthetic and natural compounds have been screened [46-48] to find effective drug candidates. However, bacteriocins have not been deeply investigated for their potent antiviral activities against SARS-CoV-2. Their pleiotropic biological effects, including the immunomodulatory effect on the human body, are known [49]. Considering their health-promoting properties, they might be an alternative source for developing therapeutics against SARS-CoV-2 [12, 50]. Indeed, bacteriocins were recently reported in few studies to be a potential therapeutic candidate for the treatment and prophylaxis of COVID-19 $[4,24,51]$.

Very few bacteriocins from probiotic bacteria were tested using in silico approaches against SARS-CoV-2 in the literature. In silico evaluation of 22 probiotic-derived bacteriocins were conducted using molecular docking and MD simulations. The RBD of S protein of SARS-CoV-2 was selected as the drug target protein because SARS-CoV-2 enters the human cells through its attachment to the ACE-2 receptor, which is a key step in infectivity with SARSCoV-2 and one of the most desired drug targets [43]. We only focused on the RBD domain of S protein because it is the key domain of S protein that binds tightly to ACE2 [31].

Protein-protein interface interactions revealed that TYR2, TYR3, THR8, LYS20, HIS38, and HIS42 are essential residues for pediocin PA-1, while TYR449, LEU455, PHE486, TYR489, GLN493, SER494, and ASN-TYR505 were key residues in the RBD of S protein. Mainly, LEU455, PHE486, and GLN493 are among the key residues for the binding affinity of S protein to ACE2 receptor. Yi et al. [46] mutated 9 amino acid residues of $S$ protein, including those three amino acids mentioned above, through single amino acid substitution mutagenesis. The binding affinity for ACE2 to the RBD was abolished upon the corresponding amino acid substitutions, indicating that they are key amino acid residues in the binding of RBD to ACE2 receptor. They also identified ALA475 and PHE486 in the SARS-CoV-2 RBD as the key amino acids for recognizing both ACE2 receptor and neutralizing antibodies, representing new binding sites for neutralizing antibodies [46]. Therefore, pediocin PA-1 could be a promising therapeutic candidate against SARS-CoV-2.

Pediocin PA-1, produced by lactic acid bacteria such as Lactococcus lactis, Pediococcus acidilactici, and Pediococcus pentosaceous, is a broad-spectrum antimicrobial agent and classified as class IIa non-lantibiotic bacteriocin [53, 54]. Mainly, it exhibits strong biological activity against Listeria monocytogenes, a foodborne pathogen and a causative agent of listeriosis [47, 48]. The mode of action for class IIa bacteriocins is based on the formation of pores in the membrane of the susceptible bacteria resulting in the dissipation of protein-motive force and the depletion of ATP [49]. In this mechanism, the bacteriocins are attracted to the target membrane receptors by electrostatic interaction due to their cationic properties [50]. On the other hand, there is no study for antiviral activity of pediocin PA-1, yet. Todorov et al. (2010) reported the antiviral activity of pediocin-like bacteriocin (99\% of DNA homology to pediocin PA-1) against herpes simplex virus [51]. This study indicated the potential antiviral activity of pediocin PA-1. Moreover, salivaricin P and B were produced by Lactobacillus salivarius and Streptococcus salivarius, respectively $[52,53]$.

Salivaricins possess selective antimicrobial activities against oral and upper respiratory tract bacteria [54]. In particular, salivaricin B is a potent antimicrobial agent with a broad-spectrum activity [55]. Salivaricins have a similar mode of action to pediocins, with an exception (salivaricin B). For instance, salivaricin 9 and salivaricin mmaye 1 form pores on the target membranes of pathogenic bacteria $[56$, 
57]. However, salivaricin $B$ does not induce pore formation in susceptible bacterial cells. Its mode of action is based on the interference with cell wall biosynthesis [52]. Additionally, the potential antiviral activity of salivaricin B for viral pharyngitis was also reported [58]. Due to the antiviral activities of bacteriocins such as peptide ST4V, enterocin CRL35, and enterocin ST5Ha, they were suggested to be potential antiviral agents in the prevention and treatment of COVID-19 [59]. Based on the current literature and our in silico findings, the most promising three bacteriocins found in the present study (pediocin PA-1, salivaricin P, and salivaricin B) can be considered as an alternative solution for the treatment and prophylaxis of SARS-CoV-2.

Biological stability and the survival of bacteriocins in the gastrointestinal tract (GIT) are also substantially crucial for considering their use as a therapeutic agent against SARS-CoV-2. Pediococcus acidilactici UL5, a producer of pediocin PA-1, indicated high resistance to GIT conditions and could produce pediocin PA-1 under GIT conditions but purified pediocin PA-1 lost the biological activity under GIT conditions. One potential solution for preventing pediocin PA-1 from GIT conditions is to be delivered in the microcapsules [60,61]. Another avenue could be developing bioactive analogs of pediocin PA-1, which can provide better biological activities and be less susceptible to GIT conditions [62]. A similar study was conducted for salivaricin $\mathrm{P}$, in which 11 analogs of salivaricin $\mathrm{P}$ were generated to prevent trypsin digestion. Eight of them were resistant to trypsin digestion while still maintaining their antimicrobial activity against $L$. monocytogenes, indicating the potential of engineered bacteriocin analogs for their biological activities and survival in GIT [63]. The biological stability studies of salivaricin B showed that it was resistant to proteolytic enzymes, such as pepsin, trypsin, and chymotrypsin, while it was partially sensitive to proteinase $\mathrm{K}$. The biological activity was stable over a range of $\mathrm{pH}$ from 3 to 9 .

Temperature stability was also tested for salivaricin $\mathrm{B}$, which was highly stable during heating and repeated freezing and thawing cycles, and was resistant to $100{ }^{\circ} \mathrm{C}$. An alternative approach for reducing the susceptibility of these promising bacteriocins against proteolytic enzymes is to engineer them to generate their analogs [62]. Another approach is to utilize those bacteriocin biosynthesizing probiotics since they might not be affected by the proteolytic enzymes in GIT, i.e., pediocin PA-1 producing probiotic [61]. Moreover, some probiotics are known to boost the immune system. For example, L. acidophilus upregulated the natural killer $\mathrm{T}$ cells in mice and played a preventive role in the influenza virus infection. In the same study, it was also found that $L$. acidophilus triggers the expression of viral defense genes in mice [65]. Probiotics can also bind to a virus and thus inhibit the binding of viral protein to the host receptor, leading to the prevention of entry of the virus [66]. In this sense, probiotics and their bacteriocins can provide a preventive role in entering viruses into the host cells. For example, Manna et al. conducted an in silico study for few bacteriocins (subtilisin, curvacin A, sakacin P, and lactoccin Gb). They found that bacteriocins had strong affinities to $S$ protein or RBD of S protein [24]. Furthermore, we performed molecular docking simulations to test how the binding energies will be affected upon bacteriocins binding to delta variant (L452R-T478K double mutant). Similar to beta variant docking results, pediocin PA-1 was found as the tightest binder compared to all studied bacteriocins (see Table S4). Therefore, it is evident that both probiotic microorganisms and their bacteriocins can be considered potential preventive therapeutics or dietary supplements to enhance the immune system for the prophylaxis of viral diseases, including COVID-19.

\section{Conclusions}

In the present study, molecular docking studies of 22 biopeptide bacteriocins that were performed against RBD of $\mathrm{S}$ protein for $\mathrm{WT}$ and beta variant revealed that pediocin PA-1 and salivaricin P had the highest binding energies to $\mathrm{WT}$ as well as mutants. Interestingly, salivaricin B also showed a strong affinity to the mutant RBDs of S protein in spite of its relatively low binding score towards WT RBD. The MD simulations confirmed the stability of bacteriocin-RBD complexes and indicated the key interactions between bacteriocins and RBDs. Mainly, pediocin PA-1 interacted with three important amino acid residues of RBD, which are among the key residues in the binding of RBD with ACE2. It is therefore clear that three bacteriocins (pediocin PA-1, salivaricin P, and salivaricin B) are the most promising therapeutic candidates with significant in silico antiviral activity against SARS-CoV-2, including beta variant. Our findings can provide a lead in further exploring bacteriocins in the treatment and prophylaxis of COVID-19. The mutations in the spike protein are evolutionarily selected for their affinity to ACE-2 and to circumvent vaccination efforts. The bacteriocins are perhaps not part of the evolutionary forces. Therefore, it is more likely that the mutation will not impair bacteriocin-S protein interaction. Although only the beta variant was studied in this work, the in silico methodology is also suitable to delta and other possible new variants of the SARS-CoV-2. By changing the amino acid sequence of the new variants on the spike protein, new structures can be prepared and used in docking and molecular dynamics simulations. By doing so, the affinity predictions of bacteriocin-S protein profiles can be predicted. 
Supplementary Information The online version contains supplementary material available at https://doi.org/10.1007/s12602-021-09879-0.

Acknowledgements All the results are in sole responsibility of the authors. The numerical calculations reported in this paper were partially performed at TUBITAK ULAKBIM, High Performance and Grid Computing Center (TRUBA resources). We also thank Mr. Huseyin Guner for kindly providing PubChem ID list of bacteriocin compounds.

Funding This work was supported by TUBITAK, 2232 - International Fellowship for Outstanding Researchers, Project number 11C244.

Data Availability The data used to support the findings of this study will be made available on reasonable request.

\section{Declarations}

Conflict of Interest The authors declare no competing interests.

\section{References}

1. Dong E, Du H, Gardner L (2020) An interactive web-based dashboard to track COVID-19 in real time. Lancet Infect Dis 20:533534. https://doi.org/10.1016/S1473-3099(20)30120-1

2. Wu F, Zhao S, Yu B et al (2020) A new coronavirus associated with human respiratory disease in China. Nature 579:265-269. https://doi.org/10.1038/s41586-020-2008-3

3. Poulter N (2020) Lower blood pressure in South Asia? Trial evidence. N Engl J Med 382:758-760. https://doi.org/10.1056/ NEJMe1917479

4. Balmeh N, Mahmoudi S, Fard NA (2021) Manipulated bio antimicrobial peptides from probiotic bacteria as proposed drugs for COVID-19 disease. Informatics Med Unlocked 23:100515. https://doi.org/10.1016/j.imu.2021.100515

5. Khodadadi E, Maroufi P, Khodadadi E et al (2020) Study of combining virtual screening and antiviral treatments of the SarsCoV-2 (Covid-19). Microb Pathog 146:104241. https://doi.org/ 10.1016/j.micpath.2020.104241

6. Hashimoto T, Perlot T, Rehman A et al (2012) ACE2 links amino acid malnutrition to microbial ecology and intestinal inflammation. Nature 487:477-481. https://doi.org/10.1038/ nature 11228

7. Zhang R, Li Y, Zhang AL et al (2020) Identifying airborne transmission as the dominant route for the spread of COVID-19. Proc Natl Acad Sci 117:14857-14863. https://doi.org/10.1073/pnas. 2009637117

8. Jin X, Lian J-S, Hu J-H et al (2020) Epidemiological, clinical and virological characteristics of 74 cases of coronavirus-infected disease 2019 (COVID-19) with gastrointestinal symptoms. Gut 69:1002-1009. https://doi.org/10.1136/gutjnl-2020-320926

9. Lin L, Jiang X, Zhang Z et al (2020) Gastrointestinal symptoms of 95 cases with SARS-CoV-2 infection. Gut 69:997-1001. https:// doi.org/10.1136/gutjnl-2020-321013

10. Ng SC, Tilg H (2020) COVID-19 and the gastrointestinal tract: more than meets the eye. Gut 69:973-974. https://doi.org/10.1136/ gutjnl-2020-321195

11. Tiwari SK, Dicks LMT, Popov IV et al (2020) Probiotics at war against viruses: what is missing from the picture? Front Microbiol 11:1877. https://doi.org/10.3389/fmicb.2020.01877

12. Akatsu H (2021) Exploring the effect of probiotics, prebiotics, and postbiotics in strengthening immune activity in the elderly. Vaccines 9:136. https://doi.org/10.3390/vaccines9020136
13. Patra S, Saxena S, Sahu N et al (2021) Systematic network and meta-analysis on the antiviral mechanisms of probiotics: a preventive and treatment strategy to mitigate SARS-CoV-2 infection. Probiotics Antimicrob Proteins 13:1138-1156. https://doi. org/10.1007/s12602-021-09748-w

14. Salminen S, Collado MC, Endo A et al (2020) The International Scientific Association of Probiotics and Prebiotics (ISAPP) consensus statement on the definition and scope of postbiotics. Nat Rev Gastroenterol Hepatol 18:649-667. https://doi.org/10.1038/ s41575-021-00440-6

15. Nataraj BH, Ali SA, Behare PV, Yadav H (2020) Postbioticsparabiotics: the new horizons in microbial biotherapy and functional foods. Microb Cell Fact 19:168. https://doi.org/10.1186/ s12934-020-01426-w

16. Tsilingiri K, Barbosa T, Penna G et al (2012) Probiotic and postbiotic activity in health and disease: comparison on a novel polarised ex-vivo organ culture model. Gut 61:1007-1015. https://doi.org/10.1136/gutjnl-2011-300971

17. Rad AH, Aghebati-Maleki L, Kafil HS et al (2021) Postbiotics, as dynamic biomolecules, and their promising role in promoting food safety. Biointerface Res Appl Chem 11:14529-14544. https://doi.org/10.33263/BRIAC116.1452914544

18. Nalbandian A, Sehgal K, Gupta A et al (2021) Post-acute COVID-19 syndrome. Nat Med 27:601-615. https://doi.org/ 10.1038/s41591-021-01283-z

19. Todorov SD, Wachsman MB, Knoetze H et al (2005) An antibacterial and antiviral peptide produced by Enterococcus mundtii ST4V isolated from soya beans. Int J Antimicrob Agents 25:508-513. https://doi.org/10.1016/j.ijantimicag.2005. 02.005

20. Férir G, Petrova MI, Andrei G et al (2013) The lantibiotic peptide labyrinthopeptin A1 demonstrates broad anti-HIV and anti-HSV activity with potential for microbicidal applications. PLoS One 8:e64010. https://doi.org/10.1371/journal.pone.0064010

21. Serkedjieva J, Danova S, Ivanova I (2000) Antiinfluenza virus activity of a bacteriocin produced by Lactobacillus delbrueckii. Appl Biochem Biotechnol 88:285-298. https://doi.org/10.1385/ ABAB:88:1-3:285

22. Quintana VM, Torres NI, Wachsman MB et al (2014) Antiherpes simplex virus type 2 activity of the antimicrobial peptide subtilosin. J Appl Microbiol 117:1253-1259. https://doi.org/10.1111/ jam. 12618

23. Anwar F, Altayb HN, Al-Abbasi FA et al (2021) Antiviral effects of probiotic metabolites on COVID-19. J Biomol Struct Dyn 39:4175-4184. https://doi.org/10.1080/07391102.2020.1775123

24. Manna S, Chowdhury T, Chakraborty R, Mandal SM (2021) Probiotics-derived peptides and their immunomodulatory molecules can play a preventive role against viral diseases including Covid-19. Probiotics Antimicrob Proteins 13:611-623. https://doi.org/10.1007/ s12602-020-09727-7

25. Lamiable A, Thévenet P, Rey J et al (2016) PEP-FOLD3: faster de novo structure prediction for linear peptides in solution and in complex. Nucleic Acids Res 44:W449-W454. https://doi.org/10. 1093/nar/gkw329

26. Salam NK, Adzhigirey M, Sherman W, Pearlman DA (2014) Structure-based approach to the prediction of disulfide bonds in proteins. Protein Eng Des Sel 27:365-374. https://doi.org/10. 1093/protein/gzu017

27. Beard H, Cholleti A, Pearlman D et al (2013) Applying physicsbased scoring to calculate free energies of binding for single amino acid mutations in protein-protein complexes. PLoS One 8:e82849. https://doi.org/10.1371/journal.pone.0082849

28. Madhavi Sastry G, Adzhigirey M, Day T et al (2013) Protein and ligand preparation: parameters, protocols, and influence on virtual screening enrichments. J Comput Aided Mol Des 27:221-234. https://doi.org/10.1007/s10822-013-9644-8 
29. Shelley JC, Cholleti A, Frye LL et al (2007) Epik: a software program for $\mathrm{pK}$ a prediction and protonation state generation for drug-like molecules. J Comput Aided Mol Des 21:681-691. https://doi.org/10.1007/s10822-007-9133-Z

30. van Zundert GCP, Rodrigues JPGLM, Trellet $M$ et al (2016) The HADDOCK2.2 web server: user-friendly integrative modeling of biomolecular complexes. J Mol Biol 428:720-725. https://doi.org/ 10.1016/j.jmb.2015.09.014

31. Wang Y, Liu M, Gao J (2020) Enhanced receptor binding of SARS-CoV-2 through networks of hydrogen-bonding and hydrophobic interactions. Proc Natl Acad Sci USA 117:13967-13974. https://doi.org/10.1073/pnas.2008209117

32. Cheng MH, Krieger JM, Kaynak B et al (2021) Impact of South African 501.V2 variant on SARS-CoV-2 spike infectivity and neutralization: a structure-based computational assessment. bioRxiv 2021.01.10.426143. https://doi.org/10.1101/2021.01.10.426143

33. Xue LC, Rodrigues JP, Kastritis PL et al (2016) PRODIGY: a web server for predicting the binding affinity of protein-protein complexes. Bioinformatics 32:btw514. https://doi.org/10.1093/ bioinformatics/btw514

34. The PyMOL Molecular Graphics System, Version 2.1 Schrödinger, LLC. https://github.com/schrodinger/pymol-open-source

35. Martyna GJ, Tobias DJ, Klein ML (1994) Constant pressure molecular dynamics algorithms. J Chem Phys 101:4177-4189. https://doi.org/10.1063/1.467468

36. Nosé $S$ (1984) A unified formulation of the constant temperature molecular dynamics methods. J Chem Phys 81:511-519. https:// doi.org/10.1063/1.447334

37. Hoover WG (1985) Canonical dynamics: equilibrium phase-space distributions. Phys Rev A Gen Phys 31:1695-1697. https://doi. org/10.1103/PhysRevA.31.1695

38. Harder E, Damm W, Maple J et al (2016) OPLS3: A force field providing broad coverage of drug-like small molecules and proteins. J Chem Theory Comput 12:281-296. https://doi.org/10. 1021/acs.jctc.5b00864

39. Jorgensen WL, Chandrasekhar J, Madura JD et al (1983) Comparison of simple potential functions for simulating liquid water. J Chem Phys 79:926-935. https://doi.org/10.1063/1.445869

40. Jacobson MP, Friesner RA, Xiang Z, Honig B (2002) On the role of the crystal environment in determining protein side-chain conformations. J Mol Biol 320:597-608. https://doi.org/10.1016/ S0022-2836(02)00470-9

41. Jacobson MP, Pincus DL, Rapp CS et al (2004) A hierarchical approach to all-atom protein loop prediction. Proteins 55(2):351367. https://doi.org/10.1002/prot.10613

42. Li J, Abel R, Zhu K, et al (2011) The VSGB 2.0 model: a next generation energy model for high resolution protein structure modeling. Proteins 79(10):2794-2812. https://doi.org/10.1002/ prot.23106

43. Humphrey W, Dalke A, Schulten K (1996) VMD: Visual molecular dynamics. J Mol Graph 14:33-38. https://doi.org/10.1016/ 0263-7855(96)00018-5

44. Abraham MJ, Murtola T, Schulz R et al (2015) GROMACS: high performance molecular simulations through multi-level parallelism from laptops to supercomputers. SoftwareX 1-2:19-25. https://doi.org/10.1016/j.softx.2015.06.001

45. Daura X, Gademann K, Jaun B et al (1999) Peptide folding: when simulation meets experiment. Angew Chemie Int Ed 38:236-240. https://doi.org/10.1002/(SICI)1521-3773(19990115)38:1/2\% 3c236::AID-ANIE236\%3e3.0.CO;2-M

46. Yi C, Sun X, Ye J et al (2020) Key residues of the receptor binding motif in the spike protein of SARS-CoV-2 that interact with ACE2 and neutralizing antibodies. Cell Mol Immunol 17:621630. https://doi.org/10.1038/s41423-020-0458-z

47. Horn N, Martínez MI, Martínez JM et al (1999) Enhanced production of pediocin PA-1 and coproduction of nisin and pediocin PA-1 by Lactococcus lactis. Appl Environ Microbiol 65:4443-4450. https://doi.org/10.1128/AEM.65.10.4443-4450. 1999

48. Rodríguez JM, Martínez MI, Kok J (2002) Pediocin PA-1, a widespectrum bacteriocin from lactic acid bacteria. Crit Rev Food Sci Nutr 42:91-121. https://doi.org/10.1080/10408690290825475

49. Chikindas ML, García-Garcerá MJ, Driessen AJ et al (1993) Pediocin PA-1, a bacteriocin from Pediococcus acidilactici PAC1.0, forms hydrophilic pores in the cytoplasmic membrane of target cells. Appl Environ Microbiol 59:3577-3584. https://doi.org/10. 1128/aem.59.11.3577-3584.1993

50. Chen Y, Ludescher RD, Montville TJ (1997) Electrostatic interactions, but not the YGNGV consensus motif, govern the binding of pediocin PA-1 and its fragments to phospholipid vesicles. Appl Environ Microbiol 63:4770-4777. https://doi.org/10.1128/aem. 63.12.4770-4777.1997

51. Todorov SD, Wachsman M, Tomé E et al (2010) Characterization of an antiviral pediocin-like bacteriocin produced by Enterococcus faecium. Food Microbiol 27:869-879. https://doi.org/10. 1016/j.fm.2010.05.001

52. Barbour A, Tagg J, Abou-Zied OK, Philip K (2016) New insights into the mode of action of the lantibiotic salivaricin B. Sci Rep 6:31749. https://doi.org/10.1038/srep31749

53. Barrett E, Hayes M, O'Connor P et al (2007) Salivaricin P, one of a family of two-component antilisterial bacteriocins produced by intestinal isolates of Lactobacillus salivarius. Appl Environ Microbiol 73:3719-3723. https://doi.org/10.1128/AEM.00666-06

54. Barbour A, Wescombe P, Smith L (2020) Evolution of lantibiotic salivaricins: new weapons to fight infectious diseases. Trends Microbiol 28:578-593. https://doi.org/10.1016/j.tim.2020.03.001

55. Hyink O, Wescombe PA, Upton M et al (2007) Salivaricin A2 and the novel lantibiotic salivaricin $b$ are encoded at adjacent loci on a 190-kilobase transmissible megaplasmid in the oral probiotic strain Streptococcus salivarius K12. Appl Environ Microbiol 73:1107-1113. https://doi.org/10.1128/AEM.02265-06

56. Barbour A, Philip K, Muniandy S (2013) enhanced production, purification, characterization and mechanism of action of salivaricin 9 lantibiotic produced by Streptococcus salivarius NU10. PLoS ONE 8:e77751. https://doi.org/10.1371/journal. pone.0077751

57. Wayah SB, Philip K (2018) Purification, characterization, mode of action, and enhanced production of Salivaricin mmaye1, a novel bacteriocin from Lactobacillus salivarius SPW1 of human gut origin. Electron J Biotechnol 35:39-47. https://doi.org/10.1016/j. ejbt.2018.08.003

58. Di Pierro F, Colombo M, Zanvit A et al (2014) Use of Streptococcus salivarius $\mathrm{K} 12$ in the prevention of streptococcal and viral pharyngotonsillitis in children. Drug Healthc Patient Saf 6:15. https://doi.org/10.2147/DHPS.S59665

59. Lee N-K, Paik H-D (2021) Prophylactic effects of probiotics on respiratory viruses including COVID-19: a review. Food Sci Biotechnol 30:773-781. https://doi.org/10.1007/s10068-021-00913-Z

60. Kheadr E, Zihler A, Dabour N et al (2010) Study of the physicochemical and biological stability of pediocin PA-1 in the upper gastrointestinal tract conditions using a dynamic in vitro model. $\mathrm{J}$ Appl Microbiol 109:54-64. https://doi.org/10.1111/j.1365-2672. 2009.04644.x

61. Fernandez B, Savard P, Fliss I (2016) Survival and metabolic activity of pediocin producer Pediococcus acidilactici UL5: its impact on intestinal microbiota and Listeria monocytogenes in a model of the human terminal ileum. Microb Ecol 72:931-942. https://doi.org/10.1007/s00248-015-0645-0

62. Bédard F, Hammami R, Zirah S et al (2018) Synthesis, antimicrobial activity and conformational analysis of the class IIa bacteriocin pediocin PA-1 and analogs thereof. Sci Rep 8:9029. https:// doi.org/10.1038/s41598-018-27225-3 
63. O'Shea EF, O'Connor PM, Cotter PD et al (2010) Synthesis of trypsin-resistant variants of the Listeria-active bacteriocin salivaricin P. Appl Environ Microbiol 76:5356-5362. https://doi.org/10. 1128/AEM.00523-10

64. Cataloluk O, Gürakan CG (2003) Characterization of salivaricin B, a protein expressed by Lactobacillus salivarius M7. Turkish J Biol 27:131-136

65. Weiss G, Rasmussen S, Zeuthen LH et al (2010) Lactobacillus acidophilus induces virus immune defense genes in murine dendritic cells by a Toll-like receptor-2-dependent mechanism.
Immunology 131:268-281. https://doi.org/10.1111/j.1365-2567. 2010.03301.x

66. Salminen S, Nybom S, Meriluoto J et al (2010) Interaction of probiotics and pathogens-benefits to human health? Curr Opin Biotechnol 21:157-167. https://doi.org/10.1016/j.copbio.2010.03. 016

Publisher's Note Springer Nature remains neutral with regard to jurisdictional claims in published maps and institutional affiliations. 